\title{
Erratum to: Quartic functional equations in Lipschitz spaces
}

\author{
Ismail Nikoufar ${ }^{1}$
}

Published online: 4 January 2016

(C) Springer-Verlag Italia 2015

\section{Erratum to: Rend Circ Mat Palermo (2015) 64:171-176 DOI 10.1007/s12215-014-0187-1}

In our original paper, we approximated the quartic functional equations in Lipschitz spaces. In the present paper, we demonstrate the results presented in our recent paper only hold for quadratic functional equations and then we correct our results and approximate the quartic functional equations in Lipschitz spaces.

\section{Introduction}

In [1] we proved the stability of quartic functional equations by using 2-variable quadratic functional equations in Lipschitz spaces. In this paper, we show that the results presented in [1] only hold for quadratic functional equations and so we correct our results and approximate the quartic functional equations by using biquadratic functional equations. Indeed, the equality $K(2 x, 2 y)=2^{4} K(x, y)$, used in [1, Theorem 2.1], does not hold for the 2-variable quadratic functional equation, that is, the function $Q$ defined in [1, Theorem 2.1] is quadratic and it is not quartic. We need to introduce the notion of symmetric left invariant mean.

Let $G$ be an abelian group, $E$ a vector space, and $S(E)$ a family of subsets of $E$. By $B(G, S(E))$ we denote the family of all functions $f: G \longrightarrow E$ such that $\operatorname{Im} f \subset A$ for some $A \in S(E)$. We say that $B(G, S(E))$ admits a symmetric left invariant mean (briefly SLIM), if the family $S(E)$ is linearly invariant and there exists a linear operator $M: B(G, S(E)) \longrightarrow E$ such that

(i) if $f_{x, y} \in B(G, S(E))$ and $(x, y) \in G \times G$, then $M\left[f_{x, y}\right]=M\left[f_{y, x}\right]$,

The online version of the original article can be found under doi:10.1007/s12215-014-0187-1.

Ismail Nikoufar

nikoufar@pnu.ac.ir

1 Department of Mathematics, Payame Noor University, P.O. Box 19395-3697, Tehran, Iran 
(ii) if $\operatorname{Im} f \subset A$ for some $A \in S(E)$, then $M[f] \in A$,

(iii) if $f \in B(G, S(E))$ and $a \in G$, then $M\left[f^{a}\right]=M[f]$,

where $f^{a}(x)=f(x+a)$. For definitions and notation we refer the readers to [1].

Czerwik et al. [2] established the stability of the following quadratic functional equation

$$
f(x+y)+f(x-y)=2 f(x)+2 f(y) .
$$

The stability type problems for some functional equations were also studied by Tabor [3,4] in Lipschitz spaces. We investigated the stability of cubic functional equations in Lipschitz spaces [5] (see also [6]). Bae [7] solved the general solution and the stability of the following 2-variable quadratic functional equation

$$
f(x+z, y+w)+f(x-z, y-w)=2 f(x, y)+2 f(z, w)
$$

in complete normed spaces. The author of the present paper investigated the stability of the quartic functional equations in Lipschitz spaces [1]. In Sect. 2, we show that our results proved in [1] hold for quadratic functional equations. In Sect. 3, we correct our results in [1] and prove the stability of quartic functional equations in Lipschitz spaces.

\section{Lipschitz stability of quadratic functional equations}

For a given function $f: G \times G \longrightarrow E$ we defined in [1] its quadratic difference as follows

$$
Q f(x, y, z, w):=2 f(x, y)+2 f(z, w)-f(x+z, y+w)-f(x-z, y-w)
$$

for all $(x, y),(z, w) \in G \times G$ and by $\Delta(G)$ we denoted the diagonal set on $G$, i.e.,

$$
\Delta(G):=\{(x, x) \in G \times G: x \in G\} .
$$

Lemma 2.1 If $f: G \times G \longrightarrow E$ is a function satisfying (1.1), then $f(2 x, 2 y)=2^{2} f(x, y)$ for all $(x, y) \in G \times G$.

Proof It is clear that $f(0,0)=0$. Set $z=x$ and $w=y$ in (1.1) to conclude the result.

Theorem 2.2 Under the hypotheses of [1, Theorem 2.1], there exists a quadratic function $Q$ such that $f_{\left.\right|_{\Delta(G)}}-Q$ is $\frac{1}{2} d$-Lipschitz.

Proof The approach of the proof is similar to that of [1, Theorem 2.1]. For every $(a, b) \in$ $G \times G$ define $F_{a, b}: G \times G \longrightarrow E$ by

$$
F_{a, b}(x, y):=\frac{1}{2} f(x+a, y+b)+\frac{1}{2} f(x-a, y-b)-f(x, y) .
$$

Set $A:=\frac{1}{2} \mathbf{d}((0,0),(a, b))+f(a, b)-f(0,0)$. Then $\operatorname{Im} F_{a, b} \subset A$ and so $F_{a, b} \in B(G \times$ $G, S(E))$. The fact that the family $B(G \times G, S(E))$ admits LIM ensures there exists a linear operator $M: B(G \times G, S(E)) \longrightarrow E$ such that

(i) $M\left[F_{a, b}\right] \in A$ for some $A \in S(E)$,

(ii) if for $(z, w) \in G \times G, F_{a, b}^{z, w}: G \times G \longrightarrow E$ is defined by $F_{a, b}^{z, w}(t, s):=F_{a, b}(t+z, s+w)$ for every $(t, s) \in G \times G$, then $F_{a, b}^{z, w} \in B(G \times G, S(E))$ and $M\left[F_{a, b}\right]=M\left[F_{a, b}^{z, w}\right]$. 
Define the function $K: G \times G \longrightarrow E$ by $K(x, y):=M\left[F_{x, y}\right]$ for $(x, y) \in G \times G$. The function $f-K$ is $\frac{1}{2} \mathbf{d}$-Lipschitz and $K$ is 2-variable quadratic. Define $Q: G \longrightarrow E$ by $Q(x):=K(x, x)$. We have

$$
f_{\left.\right|_{\Delta(G)}}-Q=f_{\left.\right|_{\Delta(G)}}-K_{\left.\right|_{\Delta(G)}}=(f-K)_{\left.\right|_{\Delta(G)}} .
$$

The function $f-K$ is $\frac{1}{2} \mathbf{d}$-Lipschitz and so is $f_{\left.\right|_{\Delta(G)}}-Q$. The following equality entails that $Q$ is quadratic.

$$
\begin{aligned}
Q(x+y)+Q(x-y) & =K(x+y, x+y)+K(x-y, x-y) \\
& =2 K(x, x)+2 K(y, y)=2 Q(x)+2 Q(y) .
\end{aligned}
$$

Theorem 2.3 Under the hypotheses of [1, Theorem 3.1], there exists a quadratic function $Q$ such that

$$
\left\|f_{\left.\right|_{\Delta(G)}}-Q\right\|_{L i p} \leq \frac{1}{2}\|Q f\|_{L i p}
$$

Proof As in the proof of [1, Theorem 3.1], define the set-valued function $\mathbf{d}:(G \times G) \times$ $(G \times G) \longrightarrow C B(E)$ by

$$
\mathbf{d}((x, y),(w, z)):=\inf _{d((x, y),(w, z)) \leq \delta} m_{Q f}(\delta) B(0,1),
$$

where $C B(E)$ is the family of all closed balls with center at zero and $B(0,1)$ is the closed unit ball with center at zero. Then, $Q f(t, s, \cdot, \cdot)$ is d-Lipschitz and so Theorem 2.2 implies there exists a quadratic function $Q$ such that $f_{\left.\right|_{\Delta(G)}}-Q$ is $\frac{1}{2} \mathbf{d}$-Lipschitz. The rest of the proof is similar to that of [1, Theorem 3.1].

\section{Lipschitz stability of quartic functional equations}

In [1] we proved the stability of quartic functional equations by using 2-variable quadratic functional equations in Lipschitz spaces. As we demonstrated in prior section the results presented in [1] only hold for quadratic functional equations. Indeed, the equality $K(2 x, 2 y)=$ $2^{4} K(x, y)$, used in [1, Theorem 2.1], does not hold for the 2-variable quadratic functional equation $K$, but such equality holds for a biquadratic functional equation (see Lemmas 2.1 and 3.2). In this section, we correct Theorems 2.1 and 3.1 of [1]

For a given function $f: G \times G \longrightarrow E$ we now define its quadratic difference as follows

$$
Q f(x, y, z):=2 f(x, z)+2 f(y, z)-f(x+y, z)-f(x-y, z)
$$

for all $x, y, z \in G$. The function $f$ is called symmetric if $f(x, y)=f(y, x)$ for all $(x, y) \in$ $G \times G$ and $f$ is called biquadratic if it is quadratic in each variable, that is,

$$
f(x+y, z)+f(x-y, z)=2 f(x, z)+2 f(y, z)
$$

and

$$
f(x, y+z)+f(x, y-z)=2 f(x, y)+2 f(x, z)
$$

for all $x, y, z \in G$. 
Consider an Abelian group $(G \times G,+)$ with a metric $d$ invariant under translation, i.e., satisfying the condition

$$
\begin{aligned}
d((x+a, y+b),(w+a, z+b)) & =d((a+x, b+y),(a+w, b+z)) \\
& =d((x, y),(w, z))
\end{aligned}
$$

for all $(a, b),(x, y),(w, z) \in G \times G$. We say that a metric $D$ on $G \times G \times G$ is a product metric if it is an invariant metric and the following condition holds

$$
\begin{aligned}
D((a, x, y),(a, w, z)) & =D((x, y, a),(w, z, a)) \\
& =d((x, y),(w, z))
\end{aligned}
$$

for all $a \in G,(x, y),(w, z) \in G \times G$.

Lemma 3.1 If $f: G \times G \longrightarrow E$ is biquadratic, then $f(0,0)=0, f(0, z)=0$, and $f(z, 0)=0$ for all $z \in G$.

Lemma 3.2 If $f: G \times G \longrightarrow E$ is biquadratic, then $f(2 x, 2 z)=2^{4} f(x, z)$ for all $(x, z) \in G \times G$.

Proof Put $x=y$ in (3.1) to obtain $f(2 x, z)=4 f(x, z)$ and set $y=z$ in (3.2) to reach $f(x, 2 z)=4 f(x, z)$ for all $(x, z) \in G \times G$. We now have

$$
f(2 x, 2 z)=4 f(x, 2 z)=4(4 f(x, z))=2^{4} f(x, z) .
$$

We now state the main results:

Theorem 3.3 Let $G$ be an Abelian group and let $E$ be a vector space. Assume that the family $B(G, S(E))$ admits SLIM. If $f: G \times G \longrightarrow E$ is a function and $Q f(t, \cdot, \cdot): G \times G \longrightarrow E$ is $\boldsymbol{d}$-Lipschitz for every $t \in G$, then there exists a quartic function $Q$ such that $f_{\left.\right|_{\Delta(G)}}-Q$ is $\frac{1}{2} d$-Lipschitz.

Proof For every $(x, y) \in G \times G$ we define $F_{x}(\cdot, y): G \longrightarrow E$ by

$$
F_{x}(\cdot, y):=\frac{1}{2} f(\cdot+x, y)+\frac{1}{2} f(\cdot-x, y)-f(\cdot, y) .
$$

We show that $F_{x}(\cdot, y) \in B(G, S(E))$. Indeed, we prove that $\operatorname{Im} F_{x}(\cdot, y) \subseteq A$ for some $A \in S(E)$. We have for $(x, y) \in G \times G$,

$$
\begin{aligned}
F_{x}(\cdot, y)= & \frac{1}{2} f(\cdot+x, y)+\frac{1}{2} f(\cdot-x, y)-f(\cdot, y)-f(x, y) \\
& -\frac{1}{2} f(\cdot, y)-\frac{1}{2} f(\cdot, y)+f(\cdot, y)+f(0, y) \\
& +f(x, y)-f(0, y) \\
= & \frac{1}{2} Q f(\cdot, x, y)-\frac{1}{2} Q f(\cdot, 0, y)+f(x, y)-f(0, y) .
\end{aligned}
$$

From the fact that $Q f(t, \cdot, \cdot)$ is $\mathbf{d}$-Lipschitz for every $t \in G$, it follows $\operatorname{Im} F_{x}(\cdot, y) \subseteq A$, where $A:=\frac{1}{2} \mathbf{d}((x, y),(0, y))+f(x, y)-f(0, y)$. The family $B(G, S(E))$ admits SLIM, so there exists a linear operator $M: B(G, S(E)) \longrightarrow E$ such that

(i) $M\left[F_{x}(\cdot, y)\right]=M\left[F_{y}(\cdot, x)\right]$ for every $(x, y) \in G \times G$, 
(ii) $M\left[F_{x}(\cdot, y)\right] \in A$ for some $A \in S(E)$ and every $(x, y) \in G \times G$,

(iii) if $z \in G$ and $F_{x}^{z}(\cdot, y): G \longrightarrow E$ defined by $F_{x}^{z}(\cdot, y):=F_{x}(\cdot+z, y)$ for every $(x, y) \in G \times G$, then $F_{x}^{z}(\cdot, y) \in B(G, S(E))$ and $M\left[F_{x}^{z}(\cdot, y)\right]=M\left[F_{x}(\cdot, y)\right]$.

Define the function $K: G \times G \longrightarrow E$ by $K(x, y):=M\left[F_{x}(\cdot, y)\right]$. It follows from property (i) of $M$ that $K$ is symmetric. By the approach as in the proof of [1, Theorem 2.1], we can show that $f-K$ is a $\frac{1}{2} \mathbf{d}$-Lipschitz function. We now have

$$
\begin{aligned}
2 K(x, y)+2 K(z, y) & =2 M\left[F_{x}(\cdot, y)\right]+2 M\left[F_{z}(\cdot, y)\right] \\
& =M\left[F_{x}^{z}(\cdot, y)\right]+M\left[F_{x}^{-z}(\cdot, y)\right]+2 M\left[F_{z}(\cdot, y)\right] .
\end{aligned}
$$

On the other hand we have

$$
\begin{aligned}
M[ & \left.F_{x}^{z}(\cdot, y)\right]+M\left[F_{x}^{-z}(\cdot, y)\right]+2 M\left[F_{z}(\cdot, y)\right] \\
= & M\left[\frac{1}{2} f(\cdot+x+z, y)+\frac{1}{2} f(\cdot-x+z, y)-f(\cdot+z, y)\right] \\
& +M\left[\frac{1}{2} f(\cdot+x-z, y)+\frac{1}{2} f(\cdot-x-z, y)-f(\cdot-z, y)\right] \\
& +M[f(\cdot+z, y)+f(\cdot-z, y)-2 f(\cdot, y)] \\
= & M\left[F_{x+z}(\cdot, y)\right]+M\left[F_{x-z}(\cdot, y)\right] \\
= & K(x+z, y)+K(x-z, y) .
\end{aligned}
$$

This shows that $K$ is quadratic on its first variable. Since $K$ is symmetric, $K$ is quadratic on its second variable and hence $K$ is biquadratic. Define $Q: G \longrightarrow E$ by $Q(x):=K(x, x)$. Then,

$$
f_{\left.\right|_{\Delta(G)}}-Q=f_{\left.\right|_{\Delta(G)}}-K_{\left.\right|_{\Delta(G)}}=(f-K)_{\left.\right|_{\Delta(G)}} .
$$

The function $f-K$ is $\frac{1}{2} \mathbf{d}$-Lipschitz and so is $f_{\left.\right|_{\Delta(G)}}-Q$. The following equality ensures that $Q$ is quartic.

$$
\begin{aligned}
Q(2 x+y)+Q(2 x-y)= & K(2 x+y, 2 x+y)+K(2 x-y, 2 x-y) \\
= & 2 K(x+y, 2 x+y)+2 K(x, 2 x+y)-K(y, 2 x+y) \\
& +2 K(x-y, 2 x-y)+2 K(x, 2 x-y)-K(y, 2 x-y) \\
= & 2[2 K(x+y, x+y)+2 K(x+y, x)-K(x+y, y)] \\
& +2[2 K(x, x+y)+2 K(x, x)-K(x, y)] \\
& -[2 K(y, x+y)+2 K(y, x)-K(y, y)] \\
& +2[2 K(x-y, x-y)+2 K(x-y, x)-K(x-y, y)] \\
& +2[2 K(x, x-y)+2 K(x, x)-K(x, y)] \\
& -[2 K(y, x-y)+2 K(y, x)-K(y, y)] \\
= & 4 K(x+y, x+y)+4 K(x-y, x-y) \\
& +8[K(x, x+y)+K(x, x-y)] \\
& -4[K(x+y, y)+K(x-y, y)] \\
& +8 K(x, x)-8 K(x, y)+2 K(y, y) \\
= & 4 K(x+y, x+y)+4 K(x-y, x-y)+24 K(x, x)-6 K(y, y) \\
= & 4 Q(x+y)+4 Q(x-y)+24 Q(x)-6 Q(y) .
\end{aligned}
$$


Theorem 3.4 Let $(G \times G,+, d, D)$ be a product metric and let $E$ be a normed space such that $B(G \times G, C B(E))$ admits SLIM. Assume that $f: G \times G \longrightarrow E$ is a function. If $Q f \in \operatorname{Lip}(G \times G \times G, E)$, then there exists a quartic function $Q$ such that

$$
\left\|f_{\left.\right|_{\Delta(G)}}-Q\right\|_{\text {Lip }} \leq \frac{1}{2}\|Q f\|_{\text {Lip }}
$$

Proof Assume that $m_{Q f}: \mathbb{R}^{+} \rightarrow \mathbb{R}^{+}$is the module of continuity of $Q f: G \times G \times G \rightarrow E$ with the product metric $D$ on $G \times G \times G$. It is immediate that

$$
\begin{aligned}
\|Q f(t, x, y)-Q f(t, w, z)\| & \leq \inf _{D((t, x, y),(t, w, z)) \leq \delta} m_{Q f}(\delta) \\
& =\inf _{d((x, y),(w, z)) \leq \delta} m_{Q f}(\delta)
\end{aligned}
$$

for all $t \in G,(x, y),(w, z) \in G \times G$. We now conclude that $Q f(t, \cdot, \cdot)$ is d-Lipschitz for the set-valued function d defined in Theorem 2.2 and so Theorem 3.3 implies there exists a quartic function $Q$ such that $f_{\left.\right|_{\Delta(G)}}-Q$ is $\frac{1}{2}$ d-Lipschitz. Using [1, Remark 2.2] with slight modifications to the notation, we get

$$
\left\|f_{\left.\right|_{\Delta(G)}}-Q\right\|_{\text {sup }} \leq \frac{1}{2}\|Q f\|_{\text {sup }}
$$

The rest of the proof is similar to that of [1, Theorem 3.1].

Acknowledgments This research was supported by a grant from Payame Noor University with title "A note on stability of quartic functional equations in Lipschitz spaces".

\section{References}

1. Nikoufar, I.: Quartic functional equations in Lipschitz spaces. Rend. Circ. Mat. Palermo 64, 171-176 (2015)

2. Czerwik, S., Dlutek, K.: Stability of the quadratic functional equation in Lipschitz spaces. J. Math. Anal. Appl. 293, 79-88 (2004)

3. Tabor, J.: Lipschitz stability of the Cauchy and Jensen equations. Results Math. 32, 133-144 (1997)

4. Tabor, J.: Superstability of the Cauchy, Jensen and isometry equations. Results Math. 35, 355-379 (1999)

5. Nikoufar, I.: Perturbation of some functional equations in Lipschitz spaces. In: 44th Annual Iranian Mathematics Conference, Mashhad (2013)

6. Ebadian, A., Ghobadipour, N., Nikoufar, I., Gordji, M.: Approximation of the cubic functional equations in Lipschitz spaces. Anal. Theory Appl. 30(4), 354-362 (2014)

7. Bae, J.-H., Park, W.-G.: A functional equation originating from quadratic forms. J. Math. Anal. Appl. 326, 1142-1148 (2007) 